\title{
Versorgungsforschung in der Allgemeinmedizin - eine bayerische Perspektive
}

\author{
Health Services Research in General Practice - A Bavarian Perspective
}

\section{Autor}

Institut

\section{A. Schneider}

Klinikum rechts der Isar, Technische Universität München, Institut für Allgemeinmedizin, München
Schlüsselwörter

- Allgemeinmedizin

- Primärversorgung

- Versorgungsforschung

\section{Key words \\ - general practice \\ - primary care \\ - health services research}

Bibliografie

DOI http://dx.doi.org/

10.1055/s-0034-1387713

Online-Publikation: 30.10.2014

Gesundheitswesen 2015;

77: 213-218

(c) Georg Thieme Verlag KG

Stuttgart · New York

ISSN 0941-3790

Korrespondenzadresse

Prof. Antonius Schneider

Klinikum rechts der Isar

Technische Universität

München

Institut für Allgemeinmedizin

Orleansstraße 47

81667 München

antonius.schneider@tum.de

\section{Zusammenfassung \\ $\nabla$}

Hintergrund: In dieser Arbeit soll ein Überblick zur allgemeinmedizinischen Versorgungsforschung in Bayern gegeben werden.

Methoden: Entwicklungslinien der allgemeinmedizinischen Versorgungsforschung in Bayern werden anhand eines narrativen Reviews aufzeigt.

Ergebnisse: Einerseits ist Versorgungsforschung ein sehr heterogener Begriff, andererseits beschreibt er die Forschungstätigkeit in der Allgemeinmedizin als „Untersuchung der letzten Meile im Gesundheitswesen" sehr zutreffend. In Bayern gibt es diesbezüglich Fortschritte; Schwerpunkte sind unter anderem Fragenstellungen zur hausärztlichen Diagnostik, Bedeutung psychosomatischer Aspekte für das Patientenmanagement und Strategien zur Sicherstellung der medizinischen Versorgung im ländlichen Raum. In Kooperation mit der Kassenärztlichen Vereinigung Bayerns wurde die Analyse von Routinedaten etabliert, was zahlreiche Möglichkeiten bietet, das Versorgungsgeschehen besser zu verstehen.

Schlussfolgerungen: Mit der zunehmenden Akademisierung der Allgemeinmedizin kommt auch die Versorgungsforschung in diesem Bereich voran. Die Versorgungsforschung sollte auch in Bayern gestärkt werden, um die aktuelle Versorgungssituation besser verstehen und effektiver gestalten zu können. Eine spezifische Herausforderung stellt das Nebeneinander von sehr großen Ballungsräumen mit hoher Arztdichte und sehr ländlichen Räumen dar. Dies gilt es sowohl im Hinblick auf die zukünftige Gestaltung der Versorgung einer immer älter werdenden Bevölkerung als auch bezüglich des drohenden Mangels an Allgemeinärzten zu berücksichtigen.

\section{Abstract \\ $\nabla$}

Background: The aim of this review is to provide an overview about health services research in Bavarian primary care.

Methods: The academic development of general practice and the associated health services research is described within this narrative review.

Results: Health services research is a heterogeneous field which might be best described for primary care as "research along the last mile of the health care System". Various elements are evolving in Bavarian primary care research, like investigation of diagnostic issues, impact of psychosomatic co-morbidity on patient management and maintenance of medical services in rural areas. Strategies to analyse and interpret routine data are established in cooperation with the Bavarian Association of Statutory Health Insurance Physicians (KVB) which alleviates understanding of health care in Bavaria.

Conclusion: The development of health services research in primary care is accompanied by the evolution of general practice as an academic discipline. Health services research should be strengthened to improve understanding and management of health care resource use. The presence of oversupply in large attractive cities combined with a shortage of physicians in rural areas is a specific challenge for health care planning in Bavaria. This is of importance for medical care of the ageing population; and needs to be addressed with reference to the shortage of general practitioners in rural areas. 


\section{Hintergrund

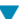

Die Versorgungsforschung ist ein heterogenes Forschungsfeld mit zahlreichen unterschiedlichen Aspekten, die von Fragestellungen zur Lebensqualität, Health Technology Assessment, Analysen von Routinedaten bis hin zu Auswirkungen von Vertragsgestaltungen, z. B. bei der Hausarztzentrierten Versorgung (HZV) reichen. Dementsprechend ist es nicht einfach, den Stand im Bereich Hausarztmedizin für Bayern - mit Vergleich zu den anderen Bundesländern - umfassend zu skizzieren. Am besten fassbar wird das Thema unter zu Hilfenahme der Definition von Pfaff, der prägnant von der „letzten Meile“ des Gesundheitswesens als Gegenstand der Versorgungsforschung spricht. Darunter ist die „konkrete Kranken- und Gesundheitsversorgung in den Krankenhäusern, Arztpraxen und sonstigen Gesundheitseinrichtungen zu verstehen“ [1,2].

Wie auch die Versorgungsforschung ist auch die Allgemeinmedizin als akademisch junge Disziplin einzuordnen. Noch im Jahr 2000 gab es insgesamt nur 5 Lehrstühle für Allgemeinmedizin an den insgesamt 36 Medizinischen Fakultäten in Deutschland. Angesichts der in den 90er Jahren zunehmenden Evidenz, dass eine gute Primärversorgung das Rückgrat von entwickelten Gesundheitssystemen darstellt $[3,4]$, da hierdurch eine sozial gerechte medizinische Versorgung gewährleistet wird, forderte der Wissenschaftsrat 1999 die Etablierung von mehr Lehrstühlen für Allgemeinmedizin an den Universitäten [5]. Mittlerweile gibt es 25 Institute oder Abteilungen mit 20 Lehrstühlen, mehrere Lehrstühle sind aktuell zur Besetzung ausgeschrieben. In Zusammenhang mit der akademischen Etablierung ist es eine Herausforderung, das Forschungsfeld der Allgemeinmedizin eindeutig zu definieren. Dies fällt deutlich schwerer als bei spezifischen Fächern wie bspw. Kardiologie oder Neurologie. Eine Hilfestellung hierzu leistet die Fachdefinition der Deutschen Gesellschaft für Allgemeinmedizin (DEGAM): „Der Arbeitsbereich der Allgemeinmedizin beinhaltet die Grundversorgung aller Patienten mit körperlichen und seelischen Gesundheitsstörungen in der Notfall-, Akut- und Langzeitversorgung sowie wesentliche Bereiche der Prävention und Rehabilitation. Allgemeinärztinnen und Allgemeinärzte sind darauf spezialisiert, als erste ärztliche Ansprechpartner bei allen Gesundheitsproblemen zu helfen. Die
Arbeitsweise der Allgemeinmedizin berücksichtigt somatische, psycho-soziale, soziokulturelle und ökologische Aspekte. Bei der Interpretation von Symptomen und Befunden ist es von besonderer Bedeutung, den Patienten, sein Krankheitskonzept, sein Umfeld und seine Geschichte zu würdigen (hermeneutisches Fallverständnis). Die Arbeitsgrundlagen der Allgemeinmedizin sind eine auf Dauer angelegte Arzt-Patienten-Beziehung und die erlebte Anamnese, die auf einer breiten Zuständigkeit und Kontinuität in der Versorgung beruhen. [6]“ Der Europäische Arm des Weltverbandes der Allgemeinärzte (World Organization of National Colleges, Academies and Academic Associations of General Practitioners/Family Physicians, WONCA) hat eine Heuristik strukturiert, mit der die Kernkompetenzen eines Hausarztes/ einer Hausärztin benannt werden [7]. Die damit verbundenen Aufgaben des Hausarztes werden mit der nachfolgenden Abbildung ( $\bullet$ Abb. 1) illustriert.

Die sich hieraus ergebenden Fragestellungen, wie diese Aspekte optimal in der Patientenversorgung realisiert werden können, sind Gegenstand der nationalen und internationalen allgemeinmedizinischen Forschung. In diesem Kontext ist allgemeinmedizinische Forschung beinahe schon Versorgungsforschung „par excellence“, da sich die entsprechenden Fragestellungen in der Regel unmittelbar daran orientieren, was „bei dem Patienten in der Praxis ankommt“. Insbesondere in Großbritannien und Holland ist in der Allgemeinmedizin eine intensive Forschungstätigkeit zu verzeichnen [8], die unter anderem auch im Feld Versorgungsforschung zu Publikationen in Top-Journals führt (siehe z. B. zuletzt $[9,10])$. Deutschland fällt - trotz deutlicher Steigerung im Output [11] - im internationalen Vergleich weit zurück [8], was unter anderem auch auf die erheblich geringeren Ressourcen für patientennahe (Versorgungs-)Forschung zurückgeführt wird [11]. Um eine Standortbestimmung zu ermöglichen, ist es Ziel dieses narrativen Reviews, einen Überblick zur allgemeinmedizinischen Versorgungsforschung in Bayern zu geben.

\section{Methodik}

$\nabla$

Es wurden die Webseiten der Institute, zugänglich über die Homepage DEGAM (www.degam.de), bezüglich aktueller Studien

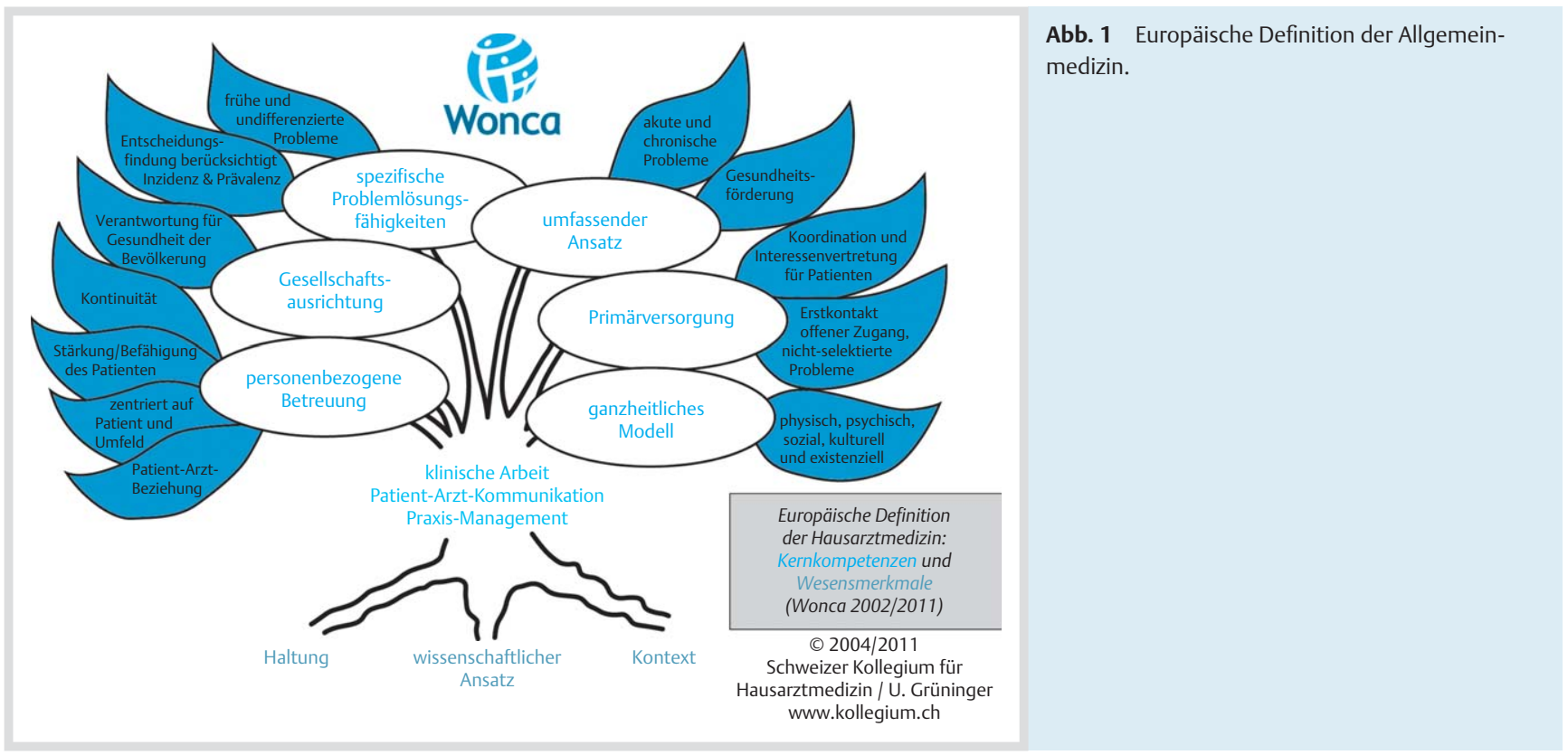


analysiert. Darüber hinaus wurden die Ergebnisse eines kürzlich am eigenen Institut durchgeführten systematischen Reviews von Originalarbeiten aus der deutschen akademischen Allgemeinmedizin verwendet [11].

\section{Diskussion der Studienlage}

In Bayern gehen die Initiativen zur Klärung von hausärztlichen Forschungsfragen im Wesentlichen vom Lehrstuhl für Allgemeinmedizin der TU München, der im Juli 2009 als Stiftungslehrstuhl der Kassenärztlichen Vereinigung Bayerns und der AOK Bayern besetzt wurde, und dem Institut der Universität Erlangen, das im September 2013 inauguriert wurde, aus. Die zentralen Aspekte der Versorgungsforschung am Institut für Allgemeinmedizin der TU München bilden diagnostische Studien, Bestimmung der Bedeutung von psychischer Komorbidität für die hausärztliche Diagnostik und Versorgungssteuerung, geriatrische Aspekte der hausärztlichen Versorgung und die Beschreibung von hausärztlicher Versorgung und Steuerungsfunktion anhand von Routinedaten. Neben diesen patientenbezogenen Studien werden zudem Untersuchungen zur Erfassung von Haltungen und Motivationslagen des medizinischen Nachwuchses durchgeführt, um hierdurch Hinweise zu erhalten, wie dem drohenden Hausärztemangel begegnet werden könnte.

\section{Diagnostische Studien}

Einen besonderen Schwerpunkt stellen am Institut für Allgemeinmedizin der TU München diagnostische Studien dar, denn die Erstellung einer korrekten Diagnose ist eine der zentralen Herausforderungen in der hausärztlichen Medizin, da sich hinter einem Symptom zahlreiche Erkrankungen verbergen können. Darüber hinaus treten die einzelnen, individuellen Erkrankungen mit einer geringen Häufigkeit in der Hausarztpraxis auf - man spricht hier vom unselektionierten Patientenklientel, in Unterscheidung zum hochselektionierten klinischen Setting [12]. Aufgrund der geringeren Prävalenz der einzelnen Erkrankungen sind in der Regel die positiven Vorhersagewerte ( = Wahrscheinlichkeit, dass jemand mit positivem Testergebnis wirklich krank ist) niedriger als im klinischen Setting [13], sodass im Niedrigprävalenzbereich des hausärztlichen Settings eine diagnostische Unschärfe resultiert. Paradigmatisch werden diese Effekte an den Krankheitsbildern Asthma bronchiale und COPD untersucht. Bspw. zeigt sich, dass die Sensitivität von Symptomen von der Primärversorgung zur Tertiärversorgung, also im klinischen Setting, zunimmt, während die Spezifität abnimmt diese Kenngrößen sind also keine fixen Größen sondern variieren in Abhängigkeit des jeweiligen Settings [14]. Aktuell wird ein vom Bundesministerium für Bildung und Forschung (BMBF) finanziertes Systematisches Review durchgeführt, in dem untersucht wird, ob exhaliertes fraktioniertes Stickstoffmonoxid eine ausreichende diagnostische Genauigkeit besitzt, um Asthma bronchiale auch ohne Bronchoprovokation zu diagnostizieren bzw. auszuschließen - dies würde die Diagnostik in der Hausarztpraxis erheblich vereinfachen. Hinweise bezüglich einer eventuellen Nutzens hierfür gab es aus einer früheren hausärztlichen Studie [15].

\section{Psychosomatische Aspekte der hausärztlichen Versorgung}

Im Kontext der Diagnosefindung müssen neben den organmedizinischen Erkrankungen auch die psychischen Aspekte berück- sichtigt werden, wenn Hausärzte den Patienten im Sinne eines ganzheitlichen bio-psycho-sozialen Ansatzes gerecht werden wollen [16]. Eine Querschnittstudie mit über 1000 Patienten aus 13 bayerischen Hausarztpraxen hat gezeigt, dass bei Patienten, bei denen der Hausarzt eine psychosomatische Dauerdiagnose vergeben hat, eine erhöhte psychische Komorbidität besteht [17] - und dass hierbei öfters Missverständnisse im Hinblick auf den aktuellen Beratungsanlass auftreten [18]. Dies ist nicht unproblematisch, denn Patienten mit einer erhöhten psychischen Komorbidität weisen eine erhöhte Inanspruchnahme von Leistungen im Gesundheitswesen auf: sie gehen häufiger zum Hausarzt, nehmen mehr Überweisungen in Anspruch und sind im Durchschnitt länger arbeitsunfähig gemeldet [19]. Dieses Inanspruchnahmeverhalten ist im Hinblick auf den Ressourcenverbrauch problematisch und potentiell gefährlich für die Patienten, da durch wiederholte diagnostische Untersuchungen (z.B. Biopsien, radiologische Untersuchungen) die Patienten geschädigt werden können. Die Bedeutung der psychischen Komorbidität für den hausärztlichen Kontext in Deutschland war bisher im Wesentlichen unklar. Eine Beschreibung der deutschen Verhältnisse ist jedoch von hoher Bedeutung, da die hausärztliche Versorgung einige Besonderheiten im Vergleich zu anderen Gesundheitssystemen aufweist. Bspw. ist die hausärztliche Kontaktrate in Deutschland außerordentlich hoch, sie liegt bei durchschnittlich 250 Arzt-Patient-Kontakten wöchentlich - in anderen Länder wie Australien, England oder Kanada ist die Kontaktrate nur halb so hoch [20]. Ursächlich hierfür ist vermutlich das quartalsbasierte Abrechnungssystem, das letztlich eine erhöhte Inanspruchnahme begünstigen könnte.

Ein weiteres Forschungsfeld betrifft das hausärztliche Verständnis der Patienten mit psychosomatischen Beschwerden oder psychischen Erkrankungen. Bspw. hat ein systematisches Review diagnostischer Studien gezeigt, dass Hausärzte eher eine psycho-soziale Sichtweise als eine rein ICD-10-orientierte Diagnostik bei depressiven Störungen bevorzugen [21]. Im Rahmen einer vom BMBF geförderten Netzwerk-Metaanalyse wird derzeit untersucht, welche therapeutischen Verfahren am effektivsten bei der Therapie von Depressionen in der Hausarztpraxis sind [22].

\section{Analyse von Routinedaten}

Die Analyse von Routinedaten ermöglicht die Beschreibung eines breiten Versorgungsgeschehens. Besondere Herausforderungen ergeben sich dadurch, dass die zu analysierenden Daten nicht zu Forschungszwecken dokumentiert werden, sondern im Rahmen von Routinetätigkeiten, also vor allem bei Abrechnungs- und Dokumentationsprozessen anfallen. Dadurch sind die Daten nicht einfach abrufbar wie bei einer Forschungsdatenbank sondern müssen entsprechend aufbereitet werden, um sie einer strukturierten Analyse zuführen zu können. Die Auswertung der Disease Management Programme (DMP) ist noch verhältnismäßig einfach, da die Dokumentation strukturiert erfolgt. Bezüglich des DMPs Asthma bronchiale sind in den ersten 4 Jahren eine Verbesserung der Pharmakotherapie und des Selbstmanagements der Patienten zu verzeichnen [23]. Dies geht mit einer Reduktion der Hospitalisierung einher. Ursächlich hierfür könnte die Notwendigkeit der Dokumentation einer evidenzbasierten Therapie, die Schulungsmaßnahmen der Kassenärztlichen Vereinigung Bayerns und die flankierende Einführung der Nationalen Versorgungsleitlinie Asthma sein, was unter Umständen zu einer Sensibilisierung beitragen hat. Die Analyse des DMP COPD zeigt ein heterogenes Bild [24]. Die Qualität der 
Versorgung scheint bezüglich einer evidenzbasierten Medikation zuzunehmen, wobei immer noch überraschend viele Patienten orales und inhalatives Kortison als Dauermedikation erhalten. Der ungünstige Krankheitsverlauf, operationalisiert anhand der stationären Notfallaufnahmen, scheint jedoch nicht aufgehalten werden zu können, was dem natürlichen Krankheitsbild der COPD, das durch eine kontinuierliche Abnahme der Lungenfunktion gekennzeichnet ist, entspricht. Andere Studien zeigen durchaus eine Reduktion von Hospitalisierung bei intensiviertem Patientenmanagement [25], sodass überlegt werden muss, wie die Versorgung von COPD-Patienten im Rahmen des DMPs weiter verbessert werden kann. Gleichzeitig zeigt die Analyse, dass Routinedaten umsichtig analysiert und mit Vorsicht interpretiert werden müssen. Drop-outs und Neuzugänge im DMP müssen dringend berücksichtigt werden, um nicht ein verzerrtes Bild der Versorgung zu erhalten. Der Fakt einer zunehmenden Hospitalisierung innerhalb der Kohorte spricht eher gegen eine Manipulation der Daten durch die betreuenden Ärzte, um ein idealistischeres Bild ihres ärztlichen Handelns zu zeichnen, als es der Realität entspricht. Es muss einschränkend festgestellt werden, dass definitive Schlussfolgerungen im Hinblick auf die Effektivität schwierig sind, da die breitflächige Einführung der DMPs keinen Vergleich mit einer Kontrollgruppe ermöglicht.

\section{Versorgung einer alternden Bevölkerung}

Insbesondere im ländlichen Raum ist eine Alterung der Bevölkerung, unter anderem aufgrund von Abwanderungen in die Städte, zu verzeichnen. Vor diesem Hintergrund muss geklärt werden, wie die Bevölkerung medizinisch weiterhin gut versorgt werden kann. Beispielhaft wurden Einwohner der oberfränkischen Gemeinde Markt Heiligenstadt, die mindestens 50 Jahre alt waren, zu ihren Wünschen für die Zukunft befragt. $81 \%$ der Befragten wohnten im Eigenheim, 90\% äußern den Wunsch, auch im Alter zu Hause zu leben und versorgt zu werden. Fast alle Teilnehmer der Befragung (98\%) haben einen Hausarzt. 17\% der Teilnehmer würden wegziehen, wenn es in der Nähe keinen Hausarzt gäbe, 6\%, wenn es keinen Facharzt und 4\%, wenn es kein Krankenhaus gäbe [26]. Am Institut wurde im Auftrag des Bayerischen Sozialministeriums untersucht, wie Pflegeeinrichtungen und niedergelassene Haus- und Fachärzte besser kooperieren können, um die Versorgung einer alternden Bevölkerung sicherzustellen. Hierbei wurde ein Kriterienkatalog für eine gelingende ärztliche Versorgung in Pflegeeinrichtungen entwickelt. Auf der Basis dieses Katalogs wird derzeit von einer Arbeitsgruppe im Sozialministerium, die sich aus Vertretern verschiedener Berufsgruppen und Behörden zusammensetzt, ein Leitfaden für die interdisziplinäre Zusammenarbeit bei der ärztlichen Versorgung von Pflegeheimbewohnern erstellt.

\section{Strategien, um dem haus- und landärztlichen Nachwuchsmangel zu begegnen}

In den nächsten Jahren ist ein erheblicher Ärztemangel im hausärztlichen Bereich, insbesondere im ländlichen Raum zu erwarten. Derzeit sind mehr als 50\% der Hausärztinnen und Hausärzte älter als 55 Jahre, sodass in den nächsten 10 Jahren mehr als die Hälfte nicht mehr in der Versorgung tätig sein wird. Dem stehen viel zu wenig Ärztinnen und Ärzte gegenüber, die den Facharzt Allgemeinmedizin erlangen. In Bayern werden derzeit jährlich maximal 200 Ärzte zur Facharztprüfung Allgemeinmedizin zugelassen, was keinesfalls ausreicht, um den Bedarf an Nachwuchs zu decken. Die Gründe hierfür sind vielschichtig. Zum einen wird eine Landflucht verantwortlich gemacht, mit der ausgedrückt wird, dass die junge Generation bevorzugt in Großstädten leben möchte. Zum anderen sinkt die Bereitschaft der Jüngeren, rund um die Uhr für die Versorgung ihrer Gemeinde zuständig zu sein. Vor diesem Hintergrund werden Einzelpraxen oft als „Auslaufmodelle“ bezeichnet. Die sogenannte „Feminisierung“ in der Medizin trägt ebenfalls zu dieser Entwicklung bei, da insbesondere Frauen auf eine gute Vereinbarkeit von Familie und Beruf achten, zunehmend jedoch auch die Männer. In einem standardisierten Fragebogen wurde die Haltung gegenüber dem Fach Allgemeinmedizin und die Bereitschaft zur Niederlassung in eigener hausärztlicher Praxis bzw. Motivation zur Arbeit in Anstellungsverhältnissen erfasst [27]. 940 (15,2\%) Studierende von insgesamt 6182 Studierenden aus 3 bayerischen Universitäten (Erlangen, LMU München und TU München) haben sich an einer internetbasierten Umfrage beteiligt. Diese Studie zeigte, dass durchaus viele Studierende gegenüber einer hausärztlichen Tätigkeit aufgeschlossen sind, allerdings präferenziell im Angestelltenverhältnis und nicht in selbstständiger Niederlassung. Eine Institutionalisierung der Allgemeinmedizin scheint bedeutsam zu sein für eine positive Einstellung zum Fach und zur Motivation für eine spätere hausärztliche Tätigkeit. Darüber hinaus sollten die Berufswünsche des ärztlichen Nachwuchses von den gesundheitspolitischen Akteuren berücksichtigt werden. Es müssen Versorgungskonzepte entwickelt werden, die auf einfacherem Weg eine Tätigkeit im Anstellungsverhältnis ermöglichen, z.B. durch hausärztliche Medizinische Versorgungszentren (MVZ). Darüber hinaus sollte überlegt werden, inwiefern Arbeitserleichterungen durch Einsatz von internetbasierten Betreuungskonzepten ermöglicht werden können. In Kooperation mit dem Deutschen Hausärzteverband wurde bspw. ein Programm entwickelt, mit dem unter hausärztlicher Koordination zahlreiche unterschiedliche Präventionsmodule umgesetzt werden können (www.hausmed.de). Die Effektivität bezüglich Gewichtsreduktion konnte in einer cluster-randomisierten Studie belegt werden [28]. Zahlreiche Einsatzmöglichkeiten von internet-basierten Technologien für die Verbesserung der Versorgung im ländlichen Raum sind hier denkbar.

\section{Weitere Aktivitäten zur allgemeinmedizinischen Versorgungsforschung in Bayern}

Da der Lehrstuhl für Allgemeinmedizin am Universitätsklinikum Erlangen erst kürzlich, im September 2013 besetzt wurde, sind dort noch keine Publikationen von Originalarbeiten zu verzeichnen. Der Ärztliche Leiter, Prof. Dr. med. Thomas Kühlein, bringt reichlich Expertise im Bereich der strukturierten Erfassung von Routinedaten mit (z.B. [29,30]), sodass diesbezüglich entsprechende Impulse zu erwarten sind. An den übrigen Medizinischen Fakultäten Bayerns sind keine allgemeinmedizinischen Lehrstühle eingerichtet. Die dort organisierten Lehrbereiche organisieren die Lehre, sind jedoch in nur geringem Maße in der Forschung aktiv. Die Kassenärztliche Vereinigung Bayerns wertet ebenfalls hausärztliche Routinedaten systematisch aus, die gelegentlich publiziert werden, in der Regel zusammen mit anderen universitären Abteilungen bzw. den Helmholtz-Instituten (z.B. $[23,24,31,32]$. Zu nennen ist noch das AOK-Präventionsprojekt INVADE, das im Landkreis Ebersberg stattfinden. Dort konnte die Wirksamkeit eines intensivierten hausärztlichen Versorgungskonzeptes zur Prävention kardiovaskulärer bzw. neurologischer Ereignisse zur Reduktion von Pflegebedürftigkeit gezeigt werden $[33,34]$. Zudem führt die Psychiatrische Klinik am Universitätsklinikum Erlangen aktuell in Kooperation mit dem Gesundheitsökonomischen Institut am Helmholtz-Zentrum 
München eine Interventionsstudie mit 129 Hausärzten und 390 Patienten durch, um Möglichkeiten zur Verbesserung der Versorgung von Patienten mit Demenz zu überprüfen. Im 2-JahresFollow-up konnten noch keine signifikanten Unterschiede zwischen einer intensivierten Betreuung und „usual care“ ermittelt werden, weitere Follow-up-Untersuchungen sind in Planung $[35,36]$.

An dieser Stelle sei auf die immanenten Limitationen eines narrativen Reviews hingewiesen. Eine orientierende Suche in Medline und Google Scholar unter der Schnittmenge „health services research“ und „Bavaria“ und („general practice“ oder „primary care“) ergab keine auskunftsreichen Treffer. Insofern musste auf den Kenntnisstand der Binnenstruktur „Forschung in der Allgemeinmedizin in Bayern“ zurückgegriffen werden, sodass die Darstellung vermutlich nicht umfassend ist.

\section{Zukünftige Herausforderungen für die allgemeinmedizinische Versorgungsforschung in Bayern}

Unter Berücksichtigung dieser Limitationen soll dennoch versucht werden, einen Ausblick auf die zukünftige Entwicklung zu geben. Die Allgemeinmedizin ist mittlerweile im akademischen Alltag angekommen [11] - dementsprechend groß ist die Fülle von Forschungsaktivitäten an der „letzten Meile im Gesundheitswesen“, sodass nur exemplarisch Beispiele aus der Versorgungsforschung dargestellt werden konnten. Der Blick auf die allgemeinmedizinische Versorgungsforschung zeigt jedoch, dass es in Bayern noch viel zu tun gibt, wenn die Versorgung für Bayern besser verstanden und gegebenenfalls optimiert werden soll. Eine spezifische Herausforderung ist das Nebeneinander von sehr großen Ballungsräumen mit hoher Arztdichte und sehr ländlichen Räumen wie Niederbayern oder Oberfranken. Denkbar ist, dass das Ausmaß an Versorgungsvariation zwischen Unter-, Über- und Fehlversorgung höher ist als in anderen Bundesländern. In diesem Zusammenhang müsste untersucht werden, wie die Organisation der haus- und landärztlichen Versorgung in Bayern verbessert werden kann. Bspw. müsste eine hausarztzentrierte Versorgung so umgesetzt werden, dass die Patienten profitieren, gleichzeitig aber auch die Arbeitsbedingungen so gestaltet werden können, dass der hausärztliche Beruf wieder für den Nachwuchs attraktiv wird. Hierzu gibt es bereits zahlreiche Ansätze, die jedoch in der Regel wenig vernetzt sind, wie z.B. Etablierung von hausärztlichen MVZ, Vergrößerung von Bereitschaftsdienstbezirken und Wiedereinführung von HZV-Verträgen. Insbesondere im Hinblick auf den letzteren Punkt wäre Begleitforschung dringend ratsam, um die Bedeutung dieser Versorgungsform besser einschätzen zu können.

Weitere wichtige Punkte betreffen die Versorgung einer immer älter werdenden Bevölkerung. Vor dem Hintergrund einer zunehmenden Multimorbidität entstehen neue Herausforderungen wie z.B. die Entwicklung neuer Gesundheitsberufe, die unter anderem in eine Akademisierung der Pflege münden. Beispiele aus dem hausärztlichen Bereich sind unter anderem die höher qualifizierte Fachangestellte VERAH ${ }^{\circledR}$, wie oben beschrieben. Diesbezüglich gilt es, diese neuen Berufsbilder und Kooperationen nicht nur zu konzeptionieren, sondern auch durch Begleitforschung weiter zu entwickeln. Darüber hinaus müssen Konzepte realisiert werden, mit der die Kooperation zwischen Pflegeeinrichtungen und niedergelassenen Ärzten ausgebaut werden kann, da der Betreuungsbedarf und der medizinische Aufwand in den nächsten Jahren erheblich steigen wird - im
Jahr 2030 kommen auf 100 Erwerbstätige im Alter von 20 bis 64 Jahren mehr als 50 Menschen über 65 Jahre, was die medizinische Versorgung vor gänzlich neuen Aufgaben stellen wird [37]. Um die Möglichkeiten zur Optimierung der Versorgung abschätzen zu können, wäre es hilfreich, die aktuelle Versorgungssituation besser zu verstehen. Ein großes Potenzial hierfür ist auch durch die Analyse von Routinedaten gegeben. Diesbezügliche Herausforderungen stellen die Zugänglichkeit zu Daten und die reduzierten Möglichkeiten zur Verknüpfung von Datenbanken dar. Strenge Datenschutzauflagen machen eine Verknüpfung von Krankenkassendaten mit Daten der Kassenärztlichen Vereinigungen oder die Kombination mit direkten Patientenbefragungen beinahe unmöglich. Darüber hinaus werden diese sogenannten Sekundärdaten nicht für Forschungszwecke strukturiert in Datenbanken erfasst, sondern müssen unter großem Aufwand vor den Analysen aufbereitet werden. Dennoch bietet die Aufbereitung der Sekundärdaten zahlreiche Möglichkeiten, um das Verständnis zum Versorgungsgeschehen zu verbessern.

\section{Fazit}

Aufgrund der Themenvielfalt ist die Versorgungsforschung in intellektueller Hinsicht ein attraktiver Bereich und kann gleichzeitig helfen, die Versorgung zu verbessern. Insbesondere für die Allgemeinmedizin zeichnet sich ein reichhaltiges Forschungsfeld ab. Eine spezifische Herausforderung für Bayern stellt das Nebeneinander von sehr großen Ballungsräumen mit hoher Arztdichte und sehr ländlichen Räumen dar. Dies gilt es sowohl im Hinblick auf die zukünftige Gestaltung der Versorgung einer immer älter werdenden Bevölkerung als auch bezüglich des drohenden Mangels an Allgemeinärzten zu berücksichtigen. Wünschenswert wäre daher eine Stärkung der Versorgungsforschung in Bayern.

Interessenkonflikt: Die Studie zur Ermittlung der Effektivität eines internet-basierten Gewichtsreduktionsprogramms wurde durch die Firma Hausmed ${ }^{\circledR}$ finanziert. Der Autor hat in diesem Zusammenhang keine persönlichen finanziellen Verbindungen. Der ursprünglich als Stiftungslehrstuhl mit Finanzierung durch AOK Bayern und KVB Bayern angelegte Lehrstuhl erhält im Rahmen der Übernahmeverhandlungen inzwischen eine Kofinanzierung durch die Medizinische Fakultät der Technischen Universität München

\section{Literatur}

1 Pfaff $H$. Versorgungsforschung - Begriffsbestimmung, Gegenstand und Aufgaben. In: Pfaff H, Schrappe M, Lauterbach K et al., Hrsg. Gesundheitsversorgung und Disease Management. Bern: Hans Huber; 2005

2 Donner-Banzhoff N, Schrappe M, Lelgemann M. Studien zur Versorgungsforschung. Eine Hilfe zur kritischen Rezeption. Z Arztl Fortbild Qualitatssich 2007; 101: 463-471

3 Starfield B. Is primary care essential? Lancet 1994; 344: 1129-1133

4 Starfield B. Primary Care: Balancing Health Needs, Services and Technology. New York: Oxford University Press; 1998

5 Wissenschaftsrat. Stellungnahme zu den Perspektiven des Faches Allgemeinmedizin an den Hochschulen 1999 http://www.wissenschafts rat.de/download/archiv/3848-98.pdf Stand: 16.01.2014

6 DEGAM. Fachdefinition der Deutschen Gesellschaft für Allgemeinmedizin http://www.degam.de/index.php?id=303 Stand: 16.01.2014

7 WONCA. The European Definition of General Practice http://www. woncaeurope.org/sites/default/files/documents/Definition \%20 3rd\%20ed\%202011\%20with\%20revised\%20wonca\%20tree.pdf Stand: 16.01.2014 
8 Glanville J, Kendrick T, McNally R et al. Research output on primary care in Australia, Canada, Germany, the Netherlands, the United Kingdom, and the United States: bibliometric analysis. BMJ 2011; 342: d1028

9 Bischoff EW, Akkermans R, Bourbeau J et al. Comprehensive self management and routine monitoring in chronic obstructive pulmonary disease patients in general practice: randomised controlled trial. BMJ 2012; 345: e7642

10 Little P, Stuart B, Francis $N$ et al. Effects of internet-based training on antibiotic prescribing rates for acute respiratory-tract infections: a multinational, cluster, randomised, factorial, controlled trial. Lancet 2013; 382: 1175-1182

11 Schneider A, Grossmann N, Linde K. The development of general practice as an academic discipline in Germany - an analysis of research output between 2000 and 2010. BMC. Fam Pract 2012; 13: 58

12 Knottnerus JA. Medical decision making by general practitioners and specialists. Fam Pract 1991; 8: 305-307

13 Schneider A, Dinant GJ, Szecsenyi J.Zur Notwendigkeit einer abgestuften Diagnostik in der Allgemeinmedizin als Konsequenz des Bayes'schen Theorems. Z Arztl Fortbild Qualitatssich 2006; 100: 121-127

14 Schneider A, Ay M, Faderl B et al. Diagnostic accuracy of clinical symptoms in obstructive airway diseases varied within different health care sectors. J Clin Epidemiol 2012; 65: 846-854

15 Schneider A, Tilemann L, Schermer T et al. Diagnosing asthma in general practice with portable exhaled nitric oxide measurement - results of a prospective diagnostic study. Respir Res 2009; 10: 15

16 Schneider A, Szecsenyi J. Psychosomatik in der Allgemeinarztpraxis ein Überblick. In: Adler RH, Herzog W, Joraschky P et al, Hrsg. Uexküll - Psychosomatische Medizin. München: Urban \& Fischer Elsevier; 2011

17 Schneider A, Hörlein E, Wartner $E$ et al. Unlimited access to health care - impact of psychosomatic co-morbidity on utilisation in German general practices. BMC. Fam Pract 2011; 12: 51

18 Schneider A, Wartner E, Schumann I et al. The impact of psychosomatic co-morbidity on discordance with respect to reasons for encounter in general practice. J Psychosom Res 2013; 74: 82-85

19 Schneider A, Hilbert B, Hörlein $E$ et al. The effect of mental comorbidity on service delivery planning in primary care: an analysis with particular reference to patients who request referral without prior assessment. Dtsch Arztebl Int 2013; 110: 653-659

20 Koch K, Miksch A, Schurmann C et al. The german health care system in international comparison: the primary care physicians' perspective. Dtsch Arztebl Int 2011; 108: 255-261

21 Schumann I, Schneider A, Kantert $C$ et al. Physicians' attitudes, diagnostic process and barriers regarding depression diagnosis in primary care: a systematic review of qualitative studies. Fam Pract 2012; 29: 255-263

22 Linde K, Schumann I, Meissner $K$ et al. Treatment of depressive disorders in primary care-protocol of a multiple treatment systematic review of randomized controlled trials. BMC Fam Pract 2011; 12: 127
23 Mehring M, Donnachie E, Mutschler R et al. Disease management programs for patients with asthma in Germany: a longitudinal population-based study. Respir Care 2013; 58: 1170-1177

24 Mehring M, Donnachie E, Fexer J et al. Disease Management Programs for Patients with chronic obstructive pulmonary disease in Germany A longitudinal evaluation of routinely collected patient records. Respir Care 2014; 59: 1123-1132

25 Lemmens KM, Nieboer AP, Huijsman R. A systematic review of integrated use of disease-management interventions in asthma and COPD. Respir Med 2009; 103: 670-691

26 Karsch-Volk $M$, Landendorfer $P$, Linde $K$ et al. Challenges for medical staff and local authorities in an aging population in a German rural area. Gesundheitswesen 2012; 74: 410-415

27 Schneider A, Karsch-Völk M, Rupp A et al. Predictors of a positive attitude of medical students towards general practice - a survey of three Bavarian medical faculties. GMS Z Med Ausbild 2013; 30: Doc45

28 Mehring $M$, Haag $M$, Linde $K$ et al. Effects of a general practice guided web-based weight reduction program - results of a cluster-randomized controlled trial. BMC. Fam Pract 2013; $14: 76$

29 Kühlein T, Laux G, Hermann $K$ et al. Krankenhauseinweisungen aus hausärztlicher Perspektive - eine deskriptive Analyse auf Basis des CONTENT-Morbiditätsregisters. Gesundheitswesen 2011; 73: 30-37

30 Kühlein T, Laux G, Gutscher A et al. Kontinuierliche Morbiditätsregistierung in der Hausarztpraxis - Vom Beratungsanlass zum Beratungsergebnis. München: Urban \& Vogel; 2008

31 Hasford J, Uricher J, Tauscher $M$ et al. Persistence with asthma treatment is low in Germany especially for controller medication - a population based study of 483,051 patients. Allergy 2010; 65: 347-354

32 Luxenhofer B, Keller M, Mielck A. Haben die Ärzte,die in ärmeren Gemeinden arbeiten,die kränkeren Diabetes-mellitus-Typ-2-Patienten? Regionale Unterschiede bei DMP-Patienten in Bayern. Diabetologie und Stoffwechsel 2009; 4: 374-383

33 Bickel H, Ander KH, Bronner M et al. Reduction of Long-Term Care Dependence After an 8-Year Primary Care Prevention Program for Stroke and Dementia: The INVADE Trial. J Am Heart Assoc 2012; 1: e000786

34 Etgen $T$, Sander $D$, Huntgeburth $U$ et al. Physical activity and incident cognitive impairment in elderly persons: the INVADE study. Arch Intern Med 2010; 170: 186-193

35 Holle R, Grassel E, Ruckdaschel $S$ et al. Dementia care initiative in primary practice: study protocol of a cluster randomized trial on dementia management in a general practice setting. BMC. Health Serv Res 2009; 9: 91

36 http://www.projekt-ida.de/media/downloads/presse/03\%20IDA\%20 Ergebnisfolder\%202\%20digital.pdf Stand: 16.01.2014

37 Sachverständigenrat zur Begutachtung der Entwicklung im Gesundheitswesen. Sondergutachten 2009: Koordination und Integration Gesundheitsversorgung in einer Gesellschaft des längeren Lebens. http://www.svr-gesundheit.de/index.php?id=14 Stand: 16.01 .2014 